
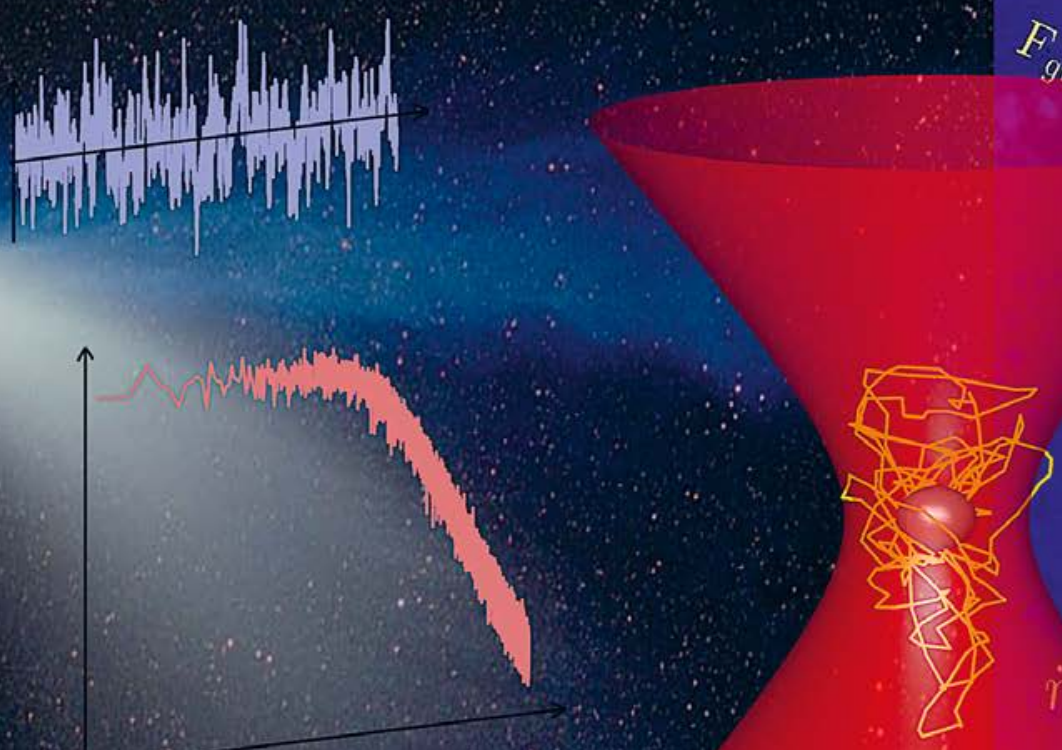

$\dot{r}(t)=\frac{F(r)}{\gamma(r)}+\sqrt{2 D(r)} W(t)$

\title{
MANIPULATING MATTER WITH LIGHT
}

I Giuseppe Pesce, Giulia Rusciano and Antonio Sasso - DOI: https://doi.org/10.1051/epn/2019202

- Università di Napoli "Federico II" - Dipartimento di Fisica "E. Pancini"

n Complesso Universitario di Monte S. Angelo - Via Cintia - 80126 Napoli, Italia

Arthur Ashkin, with his discovery of the optical tweezers, has made possible the dream of science fiction to manipulate matter with light. The optical tweezers have opened up an extremely interesting field of science, not yet exhausted, where questions of fundamental physics intertwine with intriguing investigations of biological systems at level of single macromolecule or cell.

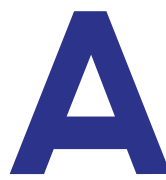
rthur Ashkin, with his discovery of the optical tweezers, has opened up an interesting research topic where fundamental physics intertwines with intriguing investigations in the biological field.

While the energetic effects of light are rather known being close to our daily life, the mechanical ones, i.e. the ability of light to exert forces and torques on the matter, are less know but much more intriguing.

The advent of the laser has made possible to use such effects for manipulating not only atoms but even objects of microscopic size. This has produced very relevant discoveries in the field of physics as proved by the numerous Nobel prizes awarded in the last two decades for laser cooling techniques and Bose-Einstein condensates. The last Nobel Prize awarded in 2018 to Arthur Ashkin "for the optical tweezers and their application to biological systems" represents a particular feature of this physics at the frontier with other scientific disciplines such as biology and medicine.

Following back the steps of this long journey quickly, it was the astronomer J. Kepler (1571-1631) to advance the first proposal of the mechanical properties of light ascribing the comets' tails formation to the "solar wind". 


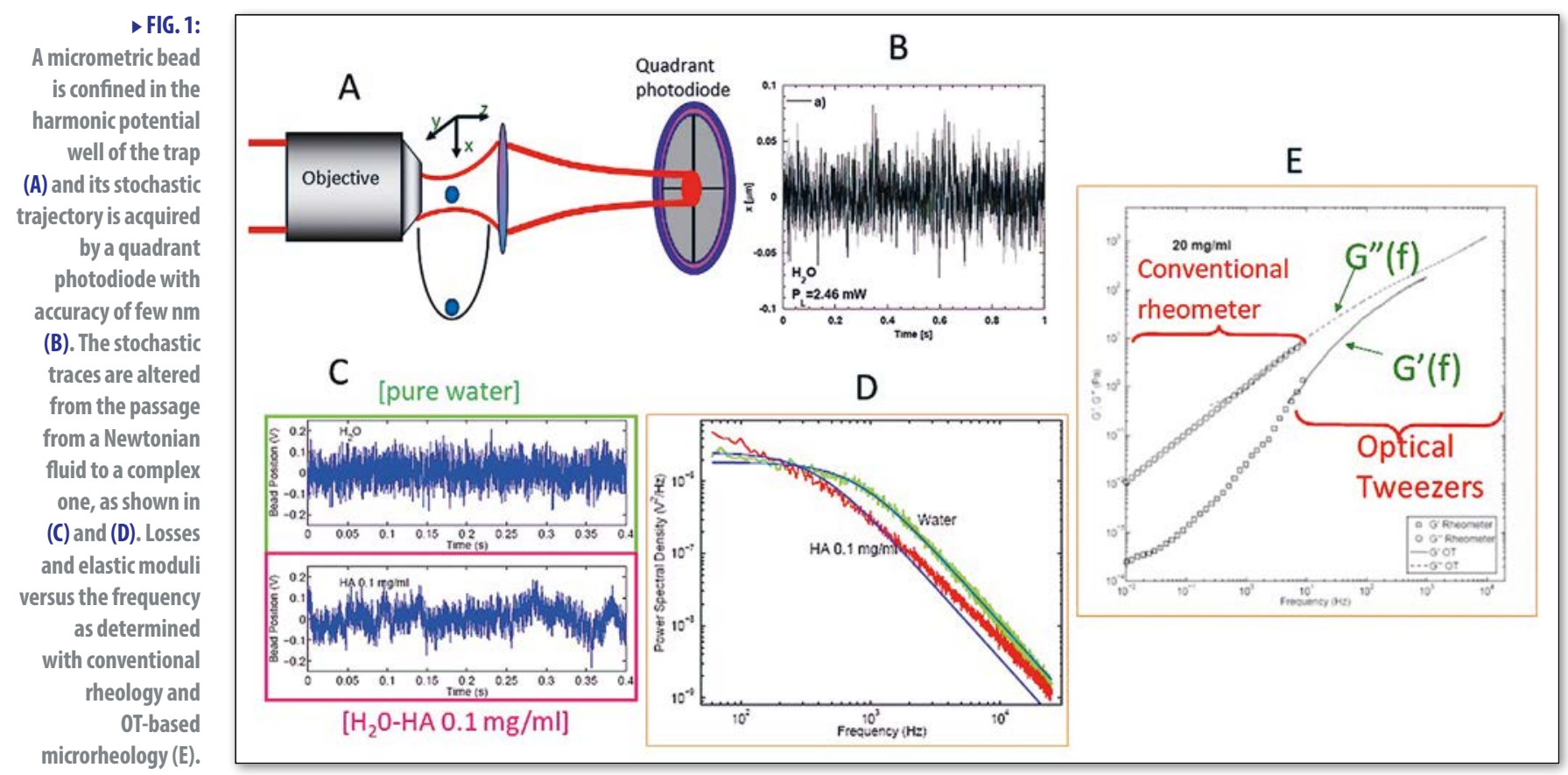

J.C. Maxwell, with his electromagnetic theory, interpreted the solar wind in terms of radiation pressure. Later on, the quantum theory introduced the concept of momentum carried per single photon.

The phenomena related to the experimental verification of the mechanical effects of light by Lebedev and Nichols and Hull (1901) using conventional lamps were certainly important but they had no follow-up for many years until the laser was invented (Maiman, 1960).

Optical manipulation, as we intend it today, is certainly due to the pioneering work of Arthur Ashkin who, using weakly focused laser beams, was able to drive and accelerate dielectric microparticles in water (1970) or to hold them suspended by balancing gravity (optical levitation). In those years he also hypothesized the possibility of rotating particles and to apply these techniques to atoms.

In 1986, Ashkin and his colleagues Dziedzic, Bjorkholm and Chu [1] realized at the Bell Labs the first optical trap or optical tweezers (OT) based on tightly focused laser beam by high-numerical aperture microscope objectives. The gradient force was so great as to overcome the radiation pressure which, instead, tends to push the particles in the direction of propagation of the laser beam. The gradient force can be approximated by $\mathrm{F}_{\mathrm{g}}=-\mathrm{k} \Delta \mathrm{x}$, i.e. it acts as a spring. Since sizes of trapped particles can range from tens of nanometers up to some tens of micrometers and considering that the involved optical forces can vary from $\mathrm{nN}$ to $\mathrm{fN}$, it was immediately evident the huge potentiality to extend OT to contactless manipulation of biological particles, as virus or cell (1987). Handling of biological particles, however, requires keeping as low as possible optical absorption to avoid thermal and photochemical damages.
From 1985 to today the number of publications based on OT has grown impressively with applications in various fields of physics and bio-medicine. In these years, the development of exotic laser beams (Bessel, Airy and Mathieu beams) based on diffractive optics and holography has led to optical lattices for multiparticle trapping for new experiments in microscopic science (particle sorting, optical binding, etc.). Shaping phase and amplitude of laser beams optical vortex have been set up for transferring orbital angular momentum. Moreover, combination of plasmonic with optical trapping has opened new possibility for bioscience at nanometric scale.

For exhaustive reviews on OT see the textbooks [2-4].

In this short article we will touch only few of the many application based on OT performed at the Department of Physics of Naples.

\section{Exploring mechanical properties of complex fluids at mesoscopic scale}

An optical trap can be approximated by a harmonic potential and a bead confined in it behaves as a sort of extremely sensitive dynamometer suitable for exploring, on a mesoscopic scale, very soft forces exchanged with environment. In water, a purely viscous medium, the confined particle is animated by Brownian motion governed by the overdamped regime Langevin equation:

$$
\dot{x}(t)=-\Gamma \frac{\mathrm{dU}}{\mathrm{dx}}+f_{\text {ther }}(t)
$$

where $1 / \Gamma=6 \pi \eta \alpha$, being $\eta$ the fluid viscosity and $\alpha$ the particle radius), $U=1 / 2 k x^{2}$ is the trap potential (with $k$ the trap stiffness $), f_{\text {ther }}(t)=\left(2 \Gamma k_{B} T\right)^{1 / 2} \delta\left(t-t^{\prime}\right)$ represents the random thermal forces. A characteristic time of the system is $t$, the ratio between the hydrodynamic factor and the trap stiffness $(\tau=1 / \Gamma k)$ which is of the order of 0.03 s. A solution of Eq.(1) can be given in terms of the mean 
square displacement (MSD) or the power spectral density (PSD) or the autocorrelation function (ACF). Therefore, once calibrated the traps, analyzing one of these functions the local viscosity around the particle can be estimated. This sort of micro-viscosimeter can also be made by revisiting the well-known Beth's experiment (1936), i.e. using spin or orbital angular momentum of the light to rotate microspheres. Balancing optical and dragging torques the particle achieves a limit angular velocity depending on the viscosity of the medium.

More intriguing is the case of complex fluids, characterized by the coexistence of viscous and elastic character. Usually such properties are measured by means of rheometers, whose main limits lie in mechanical inertia of the instruments. Moreover, even if the quantities of fluid that are needed are quite small (centiliters), in some cases, as for example for rare biological substances, there is an objective limit of their applicability. On the contrary, an OT needs only a few femtoliters and can explore viscoelasticity of environments not accessible to a conventional rheometer, even the inner part of living cell (microrheology), so allowing to study cytoplasm transformations due to external stimuli.

A spherical probe embedded in a complex fluid experiences a stochastic force $F_{x}$ and its displacement $x$ is linearly related: $x(f)=\alpha(f) F_{x}(f)$, where $\alpha(f)$ is the complex response of the material related to the complex shear modulus $G^{*}$ through the generalized Stokes-Einstein equation and the fluctuation-dissipation theorem.

In Fig. 1 is shown the essential scheme of an OT. The stochastic traces of pure water (Newtonian) and of hyaluronic-water mixtures (complex fluid) are compared in terms of the relative PSD. Only for Newtonian fluids the PSD is represented by a Lorentzian curve showing a power law $1 / \mathrm{f}^{2}$ (panel $\mathrm{D}$ ). The elastic $G^{\prime}(f)$ and viscous $G^{\prime \prime}(f)$ moduli estimated by OT well match with traditional rheometer but cover a wider frequency range [5].

\section{Molecular motors and "apparent" thermodynamics violation}

Among the many application in biology, OT have made many contributions to our understanding of the mechanochemistry of molecular motors. In 1993 Svoboda et al. [6] have studied the mechanisms which govern linear motors, as kinesin-actin, in which the energy of ATP hydrolysis in converted into mechanical work finding the characteristic steps and forces involved.

From a thermodynamics point of view, an OT behaves as a microsystem where the fluctuations of work and heat that it exchanges with the surrounding environment are comparable with thermal energy $\mathrm{k}_{\mathrm{B}} \mathrm{T}$. Hence, it is not surprising having transient violation of the second principle of thermodynamics.

Let us consider a bead dragged through water with a certain velocity $\mathrm{X}^{\prime}(\mathrm{t})$. The potential energy difference $\Delta \mathrm{U}$ between the initial and final state is $\Delta \mathrm{U}=\mathrm{W}+\mathrm{Q}$, being $\mathrm{W}$ and $\mathrm{Q}$ the thermodynamical work done on the particle and $\mathrm{Q}$ the heat exchanged by the particle with the thermal bath. The Langevin Eq. (1) can be modified by replacing the static potential $\mathrm{U}(\mathrm{x})$ into a time-dependent potential $\mathrm{U}(\mathrm{x}, \mathrm{X}(\mathrm{t}))=1 / 2 \mathrm{k}(\mathrm{x}-\mathrm{X}(\mathrm{t}))^{2}$, with $\mathrm{X}(\mathrm{t})=\mathrm{v} \cdot \mathrm{t}$. Therefore, the work done on the particle and the heat exchanged with the environment are:

$$
W=\int d X \frac{\partial \mathrm{U}}{\partial \mathrm{X}} \quad Q=\int d x \frac{\partial \mathrm{U}}{\partial \mathrm{X}}
$$

Since $\mathrm{x}$ is a stochastic variable, also the integrals of Eq.(2) are stochastic, i.e. they have to be considered in terms of probability distribution function (PDF). In our experiment [7], the work and heat done and exchanged during a single trajectory was measured by dragging the microscope stage for $5 \mathrm{~s}$ with a velocity of $1 \mu \mathrm{m} / \mathrm{s}$ repeating the procedure for 600 trajectories. In Fig. 2 it is shown the trajectory when the stage was at rest (used for trap calibration) and the successive trajectory during the motion. The estimated trapping and dragging forces were $\sim 12$ and $\sim 9 \mathrm{fN}$, respectively, hence the work done on the particle resulted actually comparable with $\mathrm{k}_{\mathrm{B}} \mathrm{T}$. The histograms and the expected PDF from theory of $W$ are plotted in Fig. 2 for times shorter $(t=0.01 \mathrm{~s})$ and longer $(t=0.5 \mathrm{~s})$ than the characteristic time $\tau=0.026 \mathrm{~s}$. For $W$ both distributions are Gaussian:
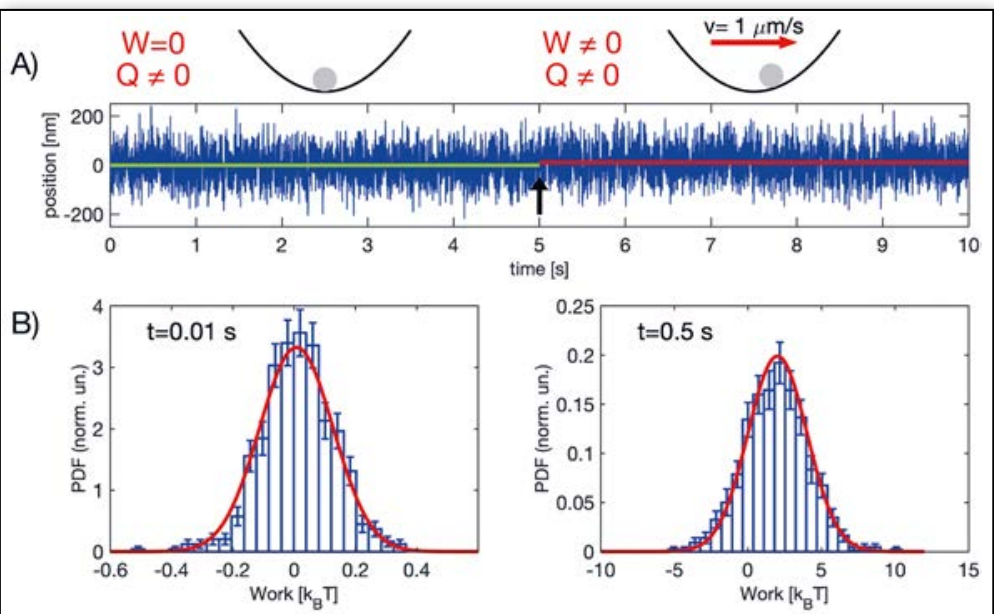

C)
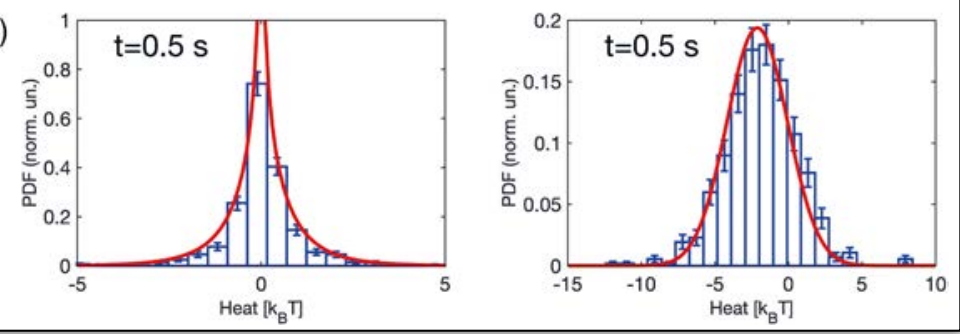

for $\mathrm{t}<<\tau$ (entropy consumption regime) the distribution is centered around zero while the peak moves to positive values as $t$ increases. The exchanged heat $\mathrm{Q}$ was instead measured: i) with the stage at rest and ii) moving the trap with $\mathrm{v}=1 \mu \mathrm{m} / \mathrm{s}$. For both cases the histograms agree with the expected theoretical PDFs; in particular the tails of the distribution were found to be exponential for the first
VFIG. 2:

(A) Stochastic trace of a trapped bead at rest and dragged at a velocity of $1 \mu \mathrm{m} / \mathrm{s}$. (B) Histograms of the work exerted on the particle by the optical trap for $t=0.01 \mathrm{~s}$ and $t=0.5 \mathrm{~s}$.

(C) Histograms of the heat exchanged by the colloidal particle with the environment, with optical trap at rest $v=0$ and with corresponds to the expected PDF as given by theory [7] with no adjustable parameter. $v=1 \mu \mathrm{m} / \mathrm{s}$. The lines 


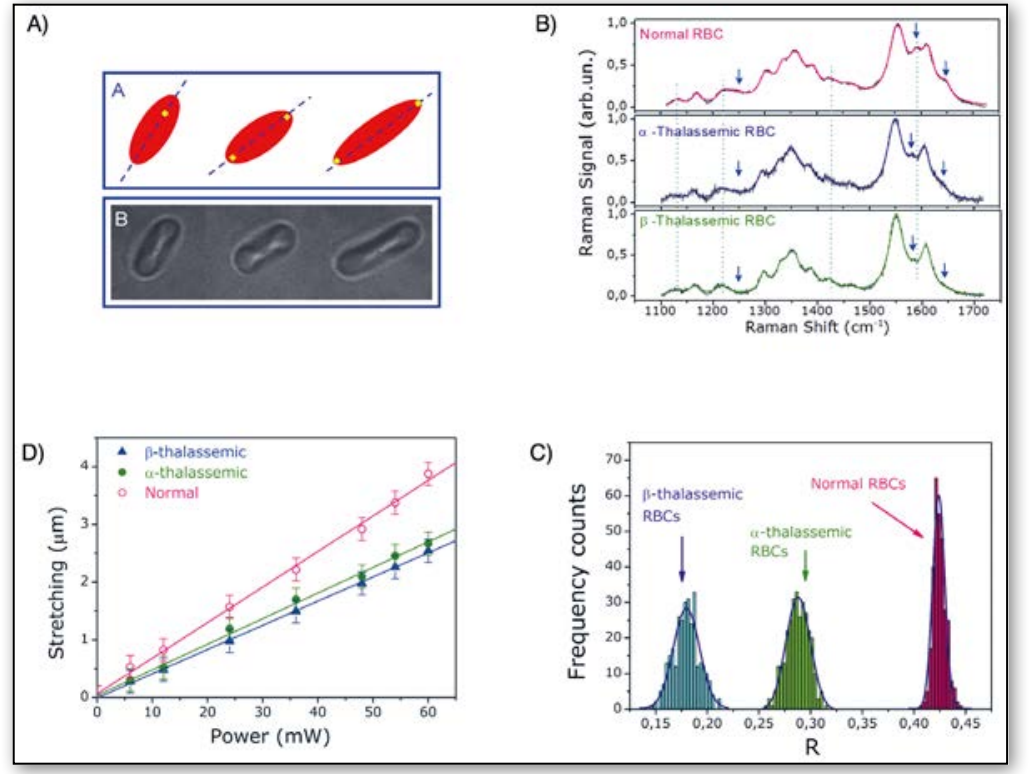

$\triangle$ FIG.3: case while the distribution resulted Gaussian for movTypical images ing trap (in agreement with theory). It can also be seen of stretched that the mean values of the work and of the heat are the $\operatorname{RBC}(A)$. Raman spectra of healthy and thalassemic erythrocytes; the two peaks highlighted by the arrows represent vibrational modes sensitive to oxy/ deoxy states (B). Histograms of the ratio $\mathrm{R}$ obtained analysing three volunteers groups

(C). Response to applied stretching

for normal and abnormal RBCs (D). negative of each other, as expected in the longtime range.

\section{Manipulation and spectroscopy of single cell}

Another significant achievement based on OT concerns their application to biological studies at the single-cell level.

By using an OT, it is possible to catch a single cell and to stretch it by trapping directly two of its ends or two bonded beads used as handles (optical stretcher).

An optical stretcher can also be realized with microfluidic systems (lab-on-a-chip) in which cells pass one at a time between two opposed, non-focused laser beams, emitted from optical fibers. These experiments allow us to study the mechanical response of a cell, a very important parameter to distinguish cells at different phase of their development, or, more importantly, healthy from diseased cells.

Optical manipulation has been also combined with Raman spectroscopy (Raman Tweezers). Raman spectroscopy is in fact able to provide a sort of digital fingerprint of the cell allowing an imaging reconstruction in terms of the cellular constituents (lipids, proteins, DNA, etc).

Red blood cells (RBCs) have been extensively investigated for this type of activity, both for the ease of finding this type of cells and for the relevance associated with many blood disorders.

For instance, one significant question is to better understand the oxy/deoxy transition of hemoglobin $\mathrm{Hb}$ ) during the stretching which occurs when erythrocytes pass through microcapillaries ( $\mathrm{Hb}$ is packaged in a smaller volume facilitating its interaction with the cell membrane in contact with the vessels). Nevertheless, alterations of oxy/deoxy states can also reflect blood diseases, including thalassemia, a genetic defect which reduces the synthesis of one of the globin chains $\alpha$ and $\beta$ of $\mathrm{Hb}$. The ratio $R=\mid v_{37} / I v_{11}$ between two $\mathrm{Hb}$ bands sensitive to iron spin-state in the heme-group, enables to distinguish quite well normal from $\alpha$ and $\beta$ thalassemic volunteers (see Fig.3).

It is interesting to note that, in addition to a reduced oxygenation capacity of the thalassemic red blood cells, the stiffness of such abnormal erythrocytes is also higher, affecting the release of oxygen during the stretching in microvessels (panel D of Fig.3) [8].

We can conclude by saying that the continued improvements of optical manipulation techniques will certainly enable many fascinating discoveries in the field of physics and biophotonics.

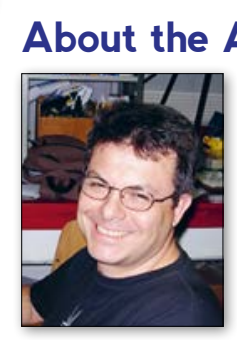

Giuseppe Pesce

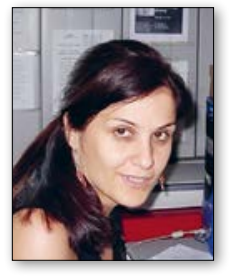

Giulia Rusciano

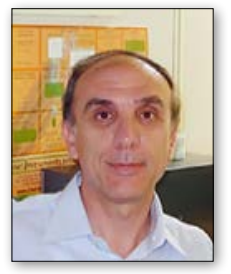

Antonio Sasso
Our group has been working with OT since 2000 dealing with many aspects related to them: force measurements, microrheology of polymeric systems and cells, applications to statistical mechanics, mechanical properties of cells. Another aspect taken care of concerns the combination of optical trapping with single cell Raman spectroscopy. More recently, the group is focused on development of Surface and Tip Enhanced Raman Spectroscopy-based systems (SERS and TERS) for the study of cell membranes.

\section{References}

[1] A. Ashkin, J. M. Dziedzic, J. E. Bjorkholm, and Steven Chu, Opt. Lett. 11, 288 (1986).

[2] A. Ashkin, Optical Trapping and Manipulation of Neutral Particles Using Lasers, World Scientific (2006)

[3] M. J. Padgett and J. Molloy, Optical Tweezers: Methods and Applications, Series in Optics and Optoelectronics, CRC Press (2010).

[4] G. Volpe, O. Maragó and P. Jones, Optical Tweezers: Principles and Applications, Cambridge University Press (2015).

[5] G. Pesce, A. C. De Luca, G. Rusciano, P. Netti, S. Fusco, A. Sasso, J. Opt. A: Pure Appl. Opt. 11, 034016 (11pp) (2009).

[6] K. Svoboda K, C.F. Schmidt, B.J.Schnapp, and S.M. Block, Nature 21, 721-7 (1993).

[7] A. Imparato, L. Peliti, G. Pesce, G. Rusciano and A. Sasso, Phys. Rev. E 76, 050101R (2007).

[8] A. C. De Luca, G. Rusciano, R. Ciancia, V. Martinelli, G. Pesce, B. Rotoli and A. Sasso, Opt. Express 16, 7943 (2008). 